\title{
Paper
}

\section{Non-linear Uniform Colour Space Considering Non-linearity and Non-symmetry in Opponent Colour Response Mechanisms}

\author{
Koichi IKEDA \\ Tokyo Rika Daigaku, Dept. Electri. Engng., Fac. Sci. Tech. \\ Received March 23, 2001
}

\begin{abstract}
A new non-linear uniform colour space NC-IIIC is developed for colour specification. First, NC-IIC space is derived by applying non-linearity at the receptor level to linear colour space NC-I composed of linear transformations of tristimulus values $X, Y$ and $Z$. Then, NC-IIIC space is constructed by further introducing non-linear and nonsymmetric functions in Y-B and R-G opponent colour response mechanisms to NC-IIC space. For colours aligned on a plane of constant value in Munsell space, coefficients of the above non-linear response functions are optimized by computer numerical analyses to make hue circles shape as close as possible to uniform circles in the new space.

The uniformity of the space is significantly improved, and the average deviation between hue circles and uniform circles in the new space is reduced to about 1/10-1/20 of those in conventional $L^{*} a^{*} b^{*}$ and $L^{*} u^{*} v^{*}$ uniform colour spaces by applying appropriate non-linear opponent transformations. Hue, lightness and chroma can be represented independently as mutually orthogonal attributes with proper correspondences of hue with hue angle, lightness with metric lightness and chroma with metric chroma in the new colour space NC-IIIC.
\end{abstract}

\section{Introduction}

The colour space is requested to satisfy the following conditions for specifying colour in accordance with a common standard

(1) Attributes of colour can be represented normally to allow mutual comparison of characteristics among colours.

(2) Specification of colour can be made in correspondence with physiological mechanisms of the visual system.

(3) Metric quantities can represent psychological aspects of colour corresponding to perceived attributes.

(4) Geometrical distance in the space is well corresponded to perceived colour difference with appropriate uniformity.

(5) Mathematical and geometrical structures are clear and lucid to assure simplicity and convenience in both calculation and specification of coordinates.

On the other hand, on the colour appearance model where chromatic adaptation is considered for the purpose of specifying how colour is appeared, the above condition (1) is different and the following conditions must be fulfilled in addition to the above statements (2) to (5).

(6) Hunt effect can be represented to show the fact that colour is more vividly perceived as illuminance level becomes higher.

(7) Stevens effect can be expressed to show the effect that colour of higher lightness against the background is perceived brighter, colour of lower lightness is perceived darker, and this effect becomes more conspicuous as the illuminance becomes higher.

(8) Helson effect can be indicated to express the phenomenon that achromatic colour of high lightness against the background is perceived closer to the colour of light source while achromatic colour of low lightness is perceived as a colour of the opposite hue of light source.

In other words, the colour appearance model should be capable of representing how colour is perceived under various circumstances, such as colour of illuminant, illuminance level, luminance and colour of the background, whether object is luminous or non-luminous, the size of the object, reflectance / luminance and colour of the object, area of visual field, viewing conditions and so forth.

Meanwhile, when specifying colour in a standard manner, achromatic colour must be represented as achromatic under any condition, and hue, lightness and chroma of the colour must be solely defined as attributes of the object regardless of illuminance level and conditions of the background.

These are the important differences between the conditions for the space to specify colour in a standard manner and those for the colour appearance model to represent how colour is appeared.

The CIE has recommended the use of $L^{*} a^{*} b^{*}$ uniform colour space and $L^{*} u^{*} V^{*}$ uniform colour space for colour specification ${ }^{11,4), 5)}$ Also, for colour rendering indices, the use of $U^{*} V^{*} W^{*}$ space was proposed.

However, these spaces did not take into consideration functions and characteristics of the opponent colour response mechanisms of the visual system and did not include equations which express non-linearity and non-symmetry corresponding with opponent responses.

Therefore, it has not been possible to represent colour accurately and uniformly in correspondence with attributes of perceived colour, such as hue lightness, and chroma ${ }^{5)}$ 
Taking the above matters into consideration, the objective of this study is to develop a new non-linear uniform colour space for the specification of colour. As the first stage, NC-II space is delivered by applying non-linearity at the receptor level to linear colour space NC-I ${ }^{6), 7)}$ which is composed through the transformation of tristimulus values $X, Y$ and $Z$.

Then final colour space NC-IIIC is developed by further introducing response functions to express non-linearity and non-symmetry in Y-B and R-G opponent colour response mechanisms.

\section{Criterion to indicate uniformity level of colour space}

On the space for colour specification, good correspondence between attributes of perceived colour and metric quantities is required. In other words, hue should correspond with hue angle or metric hue, lightness with metric lightness and chroma with distance from the origin or metric chroma, respectively, and the hue circle of constant value and constant chroma should be represented as a uniform circle on the plane of constant value.

Based on this concept, likewise the development of linear colour space NC-I with improved uniformity while maintaining linearity, Munsell colours, for which the correspondences between attributes of perceived colours and psychophysical coordinates $X, Y$ and $Z$ are defined by the Optical Society of America ${ }^{3)}$ and the Japan Industrial Standard ${ }^{2)}$, are adopted as basis for analysis. Then, the evaluation of uniformity of the colour space is conducted by estimating the relative deviation between the hue circle of colours with $V=6$ and $C=8$ and a uniform circle. The plane of $V=6$ is chosen because 1) this lightness is average in terms of perceived brightness; 2) the number of colours defined is the largest; 3) hue circles of high chroma are lined up; and 4) it is possible to review the uniformity in a wide range.

The reasons for choosing the hue circle of $C=8$ are that 1) it holds a representative position being exactly in the middle of chroma of 2 to 14; and 2) it is necessary to secure coordination with the optimization condition of linear space NC-I, which is conducted by choosing this value and chroma.

The mean radius $\langle R\rangle$ of the hue circle, that is the locus of coordinates of colours of constant value and constant chroma in the colour space which is to be evaluated, and the relative deviation $\sigma$, which indicates the relative deviation between the hue circle and a uniform circle, are expressed by the following equations.

$$
\begin{aligned}
<R> & =\sum_{\mathrm{i}}^{\mathrm{N}}=1 \sqrt{\left(X_{\mathrm{i}}^{2}+Y_{\mathrm{i}}{ }^{2}\right)} / N \\
<\Delta R\rangle^{2} & =\sum_{\mathrm{i}=1}^{\mathrm{N}}\left[\sqrt{\left(X_{\mathrm{i}}{ }^{2}+Y_{\mathrm{i}}{ }^{2}\right)}-<R>\right]^{2} / N \\
\sigma & =\langle\Delta R>/\langle R>
\end{aligned}
$$

$X_{\mathrm{i}}$ and $Y_{\mathrm{i}}$ indicate the coordinates of each colour in the space that is to be evaluated. $N$ indicates the number of colours on the hue circle. The smaller the $\sigma$, the closer the hue circle is to a uniform circle. If hue circle shapes as a uniform circle, the distance from the origin can be corresponded to chroma and hue angle to hue, and these can be expressed perpendicularly to each other.

\section{Colour space considering non-linearity at receptor level}

Here, the structuring of a colour space with improved uniformity is attempted by introducing non-linearity at the receptor level to linear colour space.

While linear colour space is generally expressed by two first-degree equations with a total of six coefficients.

By executing geometrically proper rotation of axes, one of these six coefficients can be deducted, and thus it is possible to express any linear colour space by the following two equations with five coefficients.

$$
\begin{aligned}
& a=K\left[k_{11}(X / X n)+k_{12}(Y / Y n)+k_{13}(Z / Z n)\right] \\
& b=K\left[k_{22}(Y / Y n)+k_{23}(Z / Z n)\right]
\end{aligned}
$$

To fulfill our purpose, we must select one space from these linear colour spaces for the examination. As to well-known colour spaces, there are linear space NC-I with improved linearity and a linear space adopted by Judd to express opponent colour responses of Hurvich \& Jameson.

In this study, linear colour space NC-I is chosen for the examination since it is considered appropriate to use a space of superior linearity and less distortion of hue circle.

For comparison purposes, opponent linear space of Judd is also examined supplementary.

Coordinates by which colour is specified in linear colour space NC-I and that in Judd linear space are expressed as follows. The value of $K$ is set so that the radius of hue circle with $C=8$ becomes approximately 40 .

$$
\begin{aligned}
& \text { NC-I linear space : }\left[K=154\left[8 /\left(6 L^{*} / L_{6}^{*}+2\right)\right]^{2}\right] \\
& a=K \Gamma[(X / X \mathrm{n})-[\gamma(Y / Y \mathrm{n}) \\
& +(1-\gamma)(Z / Z \mathrm{n})]] \\
& b=K \quad[(Y / Y \mathrm{n})-(Z / Z \mathrm{n})] \\
& \Gamma=2.614040 、 \gamma=0.974180 、(1-\gamma)=0.025820
\end{aligned}
$$

$$
\begin{aligned}
& \text { Judd linear space : }\left[\mathrm{K}=158\left[8 /\left(6 L^{*} / L_{6}^{*}+2\right)\right]^{2}\right] \\
& \begin{array}{l}
a=K \Gamma[(X / X \mathrm{n})-(Y / \mathrm{Yn})] \\
b=K[(Y / \mathrm{Yn})-(Z / Z \mathrm{Zn})] \\
\Gamma=2.500000: L_{6}^{*} \text { is the } L^{*} \text { corresponding to } V=6
\end{array}
\end{aligned}
$$

In the equations above, $X, Y$ and $Z$ are tristimulus values of object colour, $X \mathrm{n}, Y \mathrm{n}$ and $Z \mathrm{n}$ are tristimulus values of illuminant, and $L^{*}$ is the lightness of object colour.

In NC-I space, if $\Gamma=2.500000$ and $\gamma=1.000000$, the equations are the same as those of Judd linear space.

In this study, non-linearities concerning coordinates $a$ and $b$ relating to hue and chroma are considered, and lightness function $L$ *of the CIE is adopted in its original form, since it is structured to be well suited to brightness perception of the visual system. 

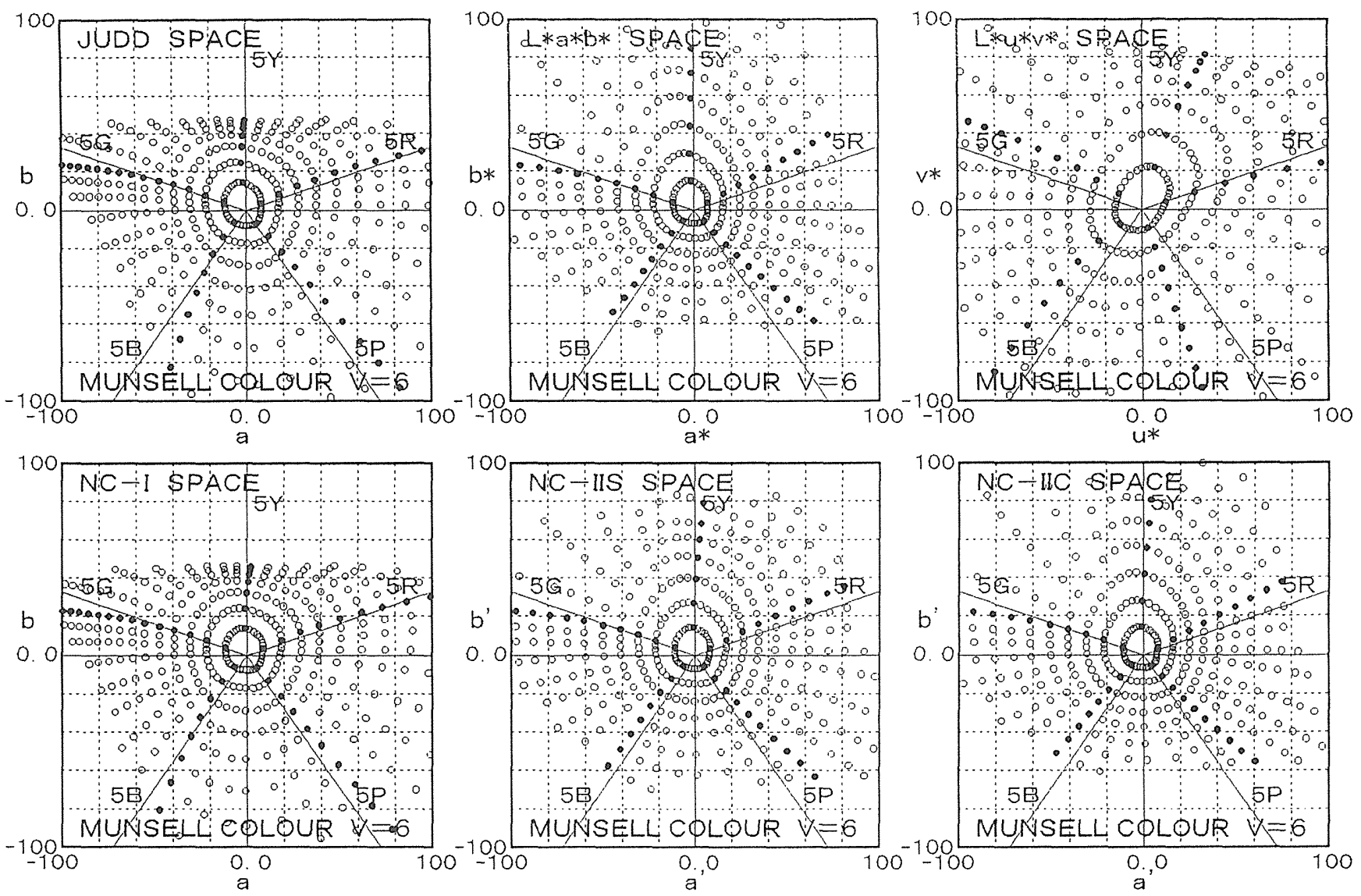

Figure 1 Coordinates of Munsell colours with value of 6 in Judd, NC- I, L*a* $b^{*}, L^{*} u^{*} v^{*}, N C-$ II $S$ and NC- II $C$ spaces

Up to this time, non-linearities at the receptor level are expressed by non-linear functions of $X, Y$ and $Z$ or $R, G$ and $B$ responses. Some of these non-linear functions, such as Munsell value that is used to express lightness, are complicated. Yet recently, approximation is attempted by employing functions that are as simple as possible, and functions of $1 / 3$ power are frequently used, for example, $L^{*} a * b *$ uniform colour space of the CIE adopts the same form of non-linear functions as well.

Non-linearity can be introduced after transforming to $R$, $G$ and $B$, however, functions of $X, Y$ and $Z$ to $1 / 3$ power are adopted in this study, because $X, Y$ and $Z$ are used in colour spaces of the CIE. For comparison purposes, further trial to study those to $1 / 2$ power is attempted.

When introducing non-linearity to linear space NC-I in conjunction with the above concept, general expression for coordinates of colour in the space is described as follows.

$$
\begin{aligned}
& L^{*}=(Y / Y n)^{1 / 3}-16 \\
& a^{\prime}=K \Gamma\left[(X / X n)^{1 / n}-\left[\gamma(Y / Y n)^{1 / n}\right.\right. \\
& \left.\left.+(1-\gamma)(Z / Z n)^{1 / n}\right]\right] \\
& b^{\prime}=K \quad\left[(Y / Y n)^{1 / n}-(Z / Z n)^{1 / n}\right]:(n=2 \text { or } 3)
\end{aligned}
$$

Also, by introducing non-linearity of $1 / 3$ power type at the receptor level to Judd linear space, the form of the expression becomes that of well-known $L^{*} a^{*} b^{*}$ uniform colour space. Yet, since this is simply for comparison purposes, only $1 / 3$ power type is applied.

$$
\begin{aligned}
& a^{*}=K \Gamma\left[\left(X / X_{n}\right)^{1 / 3}-(Y / Y n)^{1 / 3}\right] \\
& b^{*}=K \quad\left[(Y / Y n)^{1 / 3}-(Z / Z n)^{1 / 3}\right]
\end{aligned}
$$

The general term for these new colour spaces will be defined as NC-II (New Colour Space-II), and "S" (square root) and " $\mathrm{C}$ " (cubic root) will be indicated at the end for $1 / 2$ and $1 / 3$ power spaces, respectively.

Values of coefficient $K$ for respective colour spaces, by which mean radius of the hue circle of $C=8$ becomes 40 , are shown in Table 1.

Table 1 Values of $K$ in non-linear colour spaces NC- II

\begin{tabular}{|l|l|l|l|}
\hline $1 / 2$ SPACE & $\mathrm{n}=2$ & $\mathrm{NC}-$ II $\mathrm{S}$ & $\mathrm{K}=160$ \\
\hline $1 / 3$ SPACE & $\mathrm{n}=3$ & $\mathrm{NC}-$ II C & $\mathrm{K}=190$ \\
\hline $1 / 3$ SPACE & $\mathrm{n}=3$ & $\mathrm{~L} * \mathrm{a} * \mathrm{~b} *$ & $\mathrm{~K}=200$ \\
\hline
\end{tabular}

Coordinates of Munsell colours located orderly on the plane of $V=6$ in new colour spaces NC-IIS and NS-IIC are shown in Fig. 1, in comparison with those in Judd, NC-I, $L^{*} a^{*} b^{*}$ and $L^{*} u^{*} V^{*}$ colour spaces. 
It is clear from the figure that hue circles are almost symmetric in NC-I linear colour space and colour spaces NCIIS and NC-IIC, but are leaned toward the left on the top in Judd linear space and $L^{*} a * b *$ uniform colour space.

The cause of this is that Y-B response axis is tilted by shifting coordinates of $R-G$ response toward the left at the top and toward the right at the bottom. As it will be ascertained afterwards when analyzing non-linearity, this tendency becomes evident by letting $\gamma$ be 1 and coefficient associated with $Z$ be zero in equations (4) and (6).

Mean radii $\langle R\rangle$ and relative deviations $\sigma$, which express the degree of gap between hue circles and uniform circles, of hue circles of $V=6$ and $C=8$ in these colour spaces are shown in Table 2.

Table 2 Mean radii $\langle R\rangle$ and relative deviations $\sigma$ of hue circles with $V=6$ and $C=8$ in linear spaces and non-linear spaces of first kind

\begin{tabular}{|c|c|c|}
\hline COLOUR SPACE & RADIUS $<R>$ & DEVIATION $\sigma$ \\
\hline $\mathrm{Judd}$ SPACE & 40.250 & 0.0515 \\
\hline $\mathrm{L} * a * b *$ SPACE & 41.385 & 0.2280 \\
\hline $\mathrm{L} * \mathrm{u} * \mathrm{v} *$ SPACE & 57.983 & 0.1631 \\
\hline $\mathrm{NC}-\mathrm{I}$ SPACE & 40.164 & 0.0428 \\
\hline $\mathrm{NC}-$ II S SPACE & 40.299 & 0.1582 \\
\hline $\mathrm{NC}-$ II C SPACE & 40.166 & 0.2147 \\
\hline
\end{tabular}

At this stage, since non-linearity and non-symmetry in opponent colour response mechanisms are not considered, distortions of hue circles in NC-IIS and NC-IIC are instead larger than that in linear space NC-I and are almost the same as those of conventional colour spaces.

However, distortions in new spaces are slightly smaller than those of conventional spaces because the tilt of Y-B response axis is less.

\section{Non-linear and non-symmetric response function}

\subsection{Introduction of non-linear opponent function}

In opponent colour response mechanisms, it is thought that outputs from the receptors are inputted to the nervous system, where opponent colour transformations will be conducted as the next step, and that addition or subtraction will be executed to these inputs for creating Y-B and R-G opponent responses. However, there is no guarantee that these transformations or response mechanisms are linear, and that characteristics of positive and negative response outputs are symmetric.

Thus, when examining the characteristics of opponent colour response mechanisms, both non-linearity and nonsymmetry must be considered.

In this study, according to the opponent-colours theory, Y-B and R-G responses are considered to be approximately independent and orthogonal to each other, and an attempt has been made to introduce non-linearity and non-symmetry to both responses.

Equations (5) and (6) of colour spaces NC-IIS, NC-IIC and $L^{*} a{ }^{*} b^{*}$ express responses by simple addition or subtraction to / from the product of non-linear outputs from the receptors and gain constants, thus each opponent colour response in these equations is considered linear.

In other words, output $F$ is proportional to input $h$ when response is linear in the system, and the relation between these two is expressed by the following linear equation with coefficient $A$

$$
F=A h
$$

Here, $h$ is expressed by a combination of non-linear outputs of the receptors representing input to $R-G$ or $Y-B$ response.

$$
\begin{array}{r}
h \propto a^{\prime} / A \\
\text { or } \propto b^{\prime} / A
\end{array}
$$

Yet, when non-linearity lies in the response in the system, high degree terms of the input signal appear in the output equation, thus the characteristics of response can be generally expressed by a polynomial of $h$ as the following.

$$
\begin{aligned}
F & =A\left[c_{1} h+c_{2} h^{2}+c_{3} h^{3}+c_{4} h^{4}+\cdots+c_{\mathrm{P}+1} h^{\mathrm{P}+1}+\cdots\right] \\
& =A h\left[1+c_{2} h+c_{3} h^{2}+c_{4} h^{3}+\cdots+c_{\mathrm{P}+1} h^{\mathrm{P}}+\cdots\right]
\end{aligned}
$$

In the equation (9), for the purpose of locating coordinates of achromatic colour to the origin, an invariable $c_{0}$ is set to zero and $c_{1}=1$ so that output becomes zero when input is zero. On the right side of the equation, $A h$ on the left side of the brackets [ ] expresses linear response and the polynomial in the brackets expresses non-linear response.

When $c_{2}=c_{3}=c_{4}=\cdots=c_{\mathrm{P}+1}=\cdots=0$, the result of the calculation in the brackets becomes 1 , and thus the whole equation appears to be the same as that of linear response function. If non-linearity can be represented by only one high degree term, for example a degree of $\mathrm{p}+1$, then the expression will be simplified as follows.

$$
F=A h\left[1+c_{\mathrm{p}+1} h^{\mathrm{P}}\right]=A h[k]
$$

In the above equation (10), $c_{\mathrm{P}+1}$ is a coefficient showing the level of non-linearity, and $k$ in the brackets [ ] on the very right expresses non-linear response function.

\subsection{Introduction of non-symmetry to non-linear function}

A general method for introducing non-symmetry to nonlinear response is to add higher order terms to the equation (10), yet the expression by such means becomes complicated and the equation can no longer be described simply by a single non-linear term.

Generally it is known that, while positive and negative outputs of linear response are symmetric to each other 
regardless of where the centre of the response is set, however, these outputs of non-linear response can be non-symmetric by shifting the centre. Also it is known that this method is conveniently equipped with the ability to control the level of non-symmetry by adjusting the degree of shifting.

From this point of view, a coefficient $\beta$ or $1 / \beta$ is introduced, by which the shifting of the centre of non-linear response is described, and expression for non-symmetric response is attempted by substituting the variable as follows.

$$
\begin{aligned}
h & \rightarrow(h \pm \beta) \\
\text { or } & \rightarrow(h \pm 1 / \beta)= \pm(1 \pm \beta h) / \beta
\end{aligned}
$$

Considering the convenience of calculation, the latter substitution is adopted, and the coefficient of non-linearity $c_{\mathrm{P}+1}$ is replaced by the following.

$$
-\alpha= \pm c_{\mathrm{P}+1} / \beta^{\mathrm{P}}
$$

The reason for adding a minus sign before the $\alpha$ is to make the response compressed by non-linearity, or to accommodate a condition of $|k| \leqq 1$.

By making the above substitutions, response function, which describe non-linearity and non-symmetry, will be expressed as follows.

$$
F=A h\left[1-\alpha(1 \pm \beta h)^{\mathrm{P}}\right]=A h[k]
$$

In this equation, the term $A h$ on the outer left of the brackets [] on the right side shows linear response and $k$ in the brackets [ ] shows the non-linear and nonsymmetric response function.

The coefficient $\alpha$ shows the level of non-linearity, and the larger the $\alpha$ the stronger the non-linearity, and the smaller the $\alpha$ the weaker the non-linearity. When $\alpha=0$, it becomes a linear response. Also, $\beta$ is a coefficient which shows the level of non-symmetry and when $1 / \beta$ is large, or $\beta$ is small, non-symmetry is conspicuous.

\subsection{Approximation of input to opponent colour response mechanism by sine or cosine functions.}

Under the assumption that there are Y-B and R-G responses in opponent mechanisms, and that they are approximately both independent and orthogonal to each other, then opponent colour responses may be expressed in two vectors that meet at right angles.

Let the radius representing the length of a segment between the point of colour and the origin point be $R$, and let the hue angle between this segment and horizontal axis be $\theta$, then orthogonal Y-B or R-G input component will be expressed as follows.

$$
\begin{aligned}
& b^{\prime}=R \sin \theta \quad(\mathrm{X}-\mathrm{B} \text { response }) \\
& \text { or } a^{\prime}=R \cos \theta \quad \text { ( } \mathrm{R}-\mathrm{G} \text { response ) }
\end{aligned}
$$

$$
R=\sqrt{a^{\prime 2}+b^{\prime 2}}
$$

Furthermore, considering that each input signal to the opponent colour mechanism is pointing in nearly the same direction as the respective constituent, a normalized form of the above equation can be approximated as follows.

$$
\begin{aligned}
h & =b^{\prime} / R=\sin \theta & & (\mathrm{Y}-\mathrm{B} \text { input }) \\
\text { or } & =a^{\prime} / R=\cos \theta & & (\mathrm{R}-\mathrm{G} \text { input })
\end{aligned}
$$

Up to this point, the input of each opponent colour response mechanism has been considered to correspond to vertical or horizontal axis, yet there is no solid evidence that the direction of opponent colour response axis accurately match the direction of coordinate axis.

Therefore, considering that there lies a possibility that opponent colour response axis is slightly leaning away from the coordinate axis, the expression of input of Y-B or R-G opponent colour response mechanism can be revised as follows by letting the leaning angle be $\theta_{0}$.

$$
\begin{array}{rlr}
h \fallingdotseq \sin \left(\theta-\theta_{0}\right) & & (\mathrm{Y}-\mathrm{B} \text { input }) \\
\text { or } \fallingdotseq \cos \left(\theta-\theta_{0}\right) & & (\mathrm{R}-\mathrm{G} \text { input })
\end{array}
$$

Applying these approximations to the term indicating nonlinear and non-symmetric response, opponent colour response can be expressed as follows.

$$
\begin{aligned}
F & =A h[k] \\
k & =\left[1-\alpha(1 \pm \beta h)^{\mathrm{P}}\right] \\
& \fallingdotseq 1-\alpha\left[1 \pm \beta \sin \left(\theta-\theta_{0}\right)\right]^{\mathrm{p}} \\
\text { or } & \fallingdotseq 1-\alpha\left[1 \pm \beta \cos \left(\theta-\theta_{0}\right)\right]^{\mathrm{p}}
\end{aligned}
$$

In the equation above, $k$ is a function that expresses nonlinearity and non-symmetry in opponent colour response mechanism by general approximation.

\subsection{Non-linear response functions of $Y-B$ and R-G responses}

Because it is thought that non-linearity and non-symmetry exist independently in both Y-B and R-G responses, it is necessary to have two functions for such indications. However, due to non-linearity of Y-B response being remarkably larger, the analysis of this is prioritized.

In order to distinguish the above two responses, variables and function of Y-B response are marked with subscript 1 and those of $R-G$ response are marked with subscript 2.

Also, as Y-B response is almost parallel to $b^{\prime}$ axis, sine function is applied using $p$ as the exponent, and as R-G response axis is close to $a^{\prime}$ axis, cosine function is adopted using exponent $q$. Moreover, the sign of coefficient $\beta$ to express the direction of shift of the centre is decided with consideration of the fact that the level of non-linearity is larger in the direction of $\mathrm{Y}$ for $\mathrm{Y}-\mathrm{B}$ response and that of $\mathrm{G}$ for $\mathrm{R}-\mathrm{G}$ response. Non-linear functions to express non-linearity 
and non-symmetry for both responses will be as follows.

$$
\begin{array}{ll}
k_{1}=1-\alpha_{1}\left[1+\beta_{1} \sin \left(\theta-\theta_{1}\right)\right]^{\mathrm{p}} & (\mathrm{Y}-\mathrm{B} \text { non-linear }) \\
k_{2}=1-\alpha_{2}\left[1-\beta_{2} \cos \left(\theta-\theta_{2}\right)\right]^{\mathrm{q}} & (\mathrm{R}-\mathrm{G} \text { non-linear })
\end{array}
$$

At this point, both non-linear and non-symmetric responses should be obtained by multiplying the above functions to Y-B and R-G responses, respectively. However, geometrically inconvenient condition that hue angle will not be the same before and after obtaining the responses of nonlinearity and non-symmetry will occur as shown below.

$$
\tan ^{-1}\left(b^{\prime} / a^{\prime}\right) \neq \tan ^{-1}\left(b^{\prime} k_{1} / a^{\prime} k_{2}\right)
$$

In order to avoid such inconvenience, the measure taken is to multiply respective responses by both functions as shown next equation so that hue angle would not be changed

$$
F=A h\left[k_{1}\right]\left[k_{2}\right]
$$

By doing so, one function may influence the other response. Yet if the characteristics of the following are considered, the other supplementary function will not bring non-symmetric influence to the other response and non-linear influence will not occur to the response on the other axis.

$$
\begin{array}{ll}
\sin \left(\theta_{c}\right)=\sin \left(180^{\circ}-\theta_{c}\right) & (\mathrm{Y}-\mathrm{B} \text { axis reversal symmetry) } \\
\cos \left(\theta_{\mathrm{c}}\right)=\cos \left(-\theta_{\mathrm{c}}\right) & (\mathrm{R}-\mathrm{G} \text { axis reversal symmetry) } \\
\theta_{\mathrm{c}}=0^{\circ} \text { or } 180^{\circ} \sin \left(\theta_{\mathrm{c}}\right)=0 & \text { (non-linear term zero on } \mathrm{R}-\mathrm{G}) \\
\theta_{\mathrm{c}}=90^{\circ} \text { or } 270^{\circ} \cos \left(\theta_{\mathrm{c}}\right)=0 & \text { (non-linear term zero on } \mathrm{Y}-\mathrm{B}) \\
\theta_{\mathrm{c}}=\theta-\theta_{0} & (21)
\end{array}
$$

Therefore, generally speaking, the impact is minimal and it will not be a problem in terms of practical use by applying this idea.

At this stage, the components $a^{\prime}$ and $b^{\prime}$ which are responses prior to the introduction of non-linearity in opponent mechanisms in colour space NC-IIS or NC-IIC are applied as an approximation for the term $A h$ indicating linear response in the expression for new colour space.

Then, collecting above ideas and arranging them in order, coordinates of at and $b+$ in new colour space, in which non-linear and non-symmetric responses in opponent mechanisms are considered, will be expressed as follows.

$$
\begin{aligned}
& A=R \Rightarrow A h=a^{\prime} \text { or } b^{\prime} \\
& a \dagger=A h\left[k_{1}\right]\left[k_{2}\right]=k_{1} k_{2} a^{\prime} \\
& b \dagger=A h\left[k_{1}\right]\left[k_{2}\right]=k_{1} k_{2} b^{\prime} \\
& a^{\prime}=K \Gamma\left[(X / X n)^{1 / \mathrm{n}}-\left[\gamma(Y / Y n)^{1 / \mathrm{n}}\right.\right. \\
& \left.\left.\quad+(1-\gamma)(Z / Z n)^{1 / \mathrm{n}}\right]\right] \\
& b^{\prime}=K\left[(Y / Y n)^{1 / \mathrm{n}}-(Z / Z n)^{1 / \mathrm{n}}\right] \quad(\mathrm{n}=2 \text { or } 3) \\
& k_{1}=1-\alpha_{1}\left[1+\beta_{1} \sin \left(\theta-\theta_{1}\right)\right]^{\mathrm{p}} \\
& k_{2}=1-\alpha_{2}\left[1-\beta_{2} \cos \left(\theta-\theta_{2}\right)\right]^{\mathrm{q}} \\
& \theta=\tan ^{-1}\left(b^{\prime} / a^{\prime}\right)=\tan ^{-1}\left(b \dagger / a^{\dagger}\right)
\end{aligned}
$$

Here, $a^{\prime}$ and $b^{\prime}$ are colour coordinates in colour space NC-IIS or NC-IIC as expressed in equation (5). In addition, constant $K$ is to be adjusted accordingly to make the radius of the hue circle approximately 40 .

\section{Non-linear and non-symmetric uniform colour space} 5.1 Coefficients for non-linear and non-symmetric functions

Non-linear and non-symmetric opponent colour response functions are tried to introduce to the expressions for NC-IIS, NC-IIC and $L^{*} a^{*} b^{*}$ colour spaces. Optimum response functions are searched by computer analysis so as to make the hue circle shape as close as possible to a uniform circle.

Computer numerical analysis has been proceeded, by changing exponent $\mathrm{p}$ of response function $k_{1}$ and coefficients $\alpha_{1}, \beta_{1}$, and $\theta_{1}$, and exponent $\mathrm{q}$ of $k_{2}$ and coefficients $\alpha_{2}, \beta_{2}$, and $\theta_{2}$ in sequence, in order to minimize the relative deviation $\sigma$ which shows the level of gap between the hue circle and a uniform circle.

Meanwhile, because it is preferable that exponents $\mathrm{p}$ and $\mathrm{q}$ be small, calculations have been conducted in order starting from the lowest degree. However, both $k_{1}$ and $k_{2}$ must be degrees of even numbers due to their symmetric characteristics that they compress high chroma parts of both positive and negative directions. Therefore, the above exponents have been changed in the order of $2,4,6,8$, and the appropriate numbers for the degree, which minimize relative deviation, are adopted.

Analysis has been conducted by first setting the indices in due order, and secondly executing test calculations by changing coefficients over a wide range. Fortunately, there is only one area in which the set of coefficients can make relative deviation $\sigma$ the local minimum. In other words, as there are no multiple local minimums, there is no risk of straying into a local minimum which is not the absolute minimum. Therefore, it is found that the minimum can be obtained by changing coefficients in turn so that the local minimum $\sigma$ becomes smaller.

Through the above, the respective coefficients of the area that is close to the area proven to have the minimum in the initial research, are adopted as initial values. Then coefficients are selected and changed in order to seek the values that make $\sigma$ the local minimum.

After all coefficients have been tested the same analysis is conducted again in the same order starting from the resulted values of coefficients. This procedure has been repeated time after time until the finding converged to the absolute minimum, in other words, to obtain optimum solution.

As representative examples of the procedure of numerical analysis when changing respective coefficients of non-linear response functions in order, the changes of relative deviation $\sigma$ corresponding to coefficients on $1 / 3$ power space NC-IIIC are shown in Fig. 2. The changes of $\sigma$ are almost the same in other respective colour spaces.

As a result of numerical analysis, the optimum solutions 

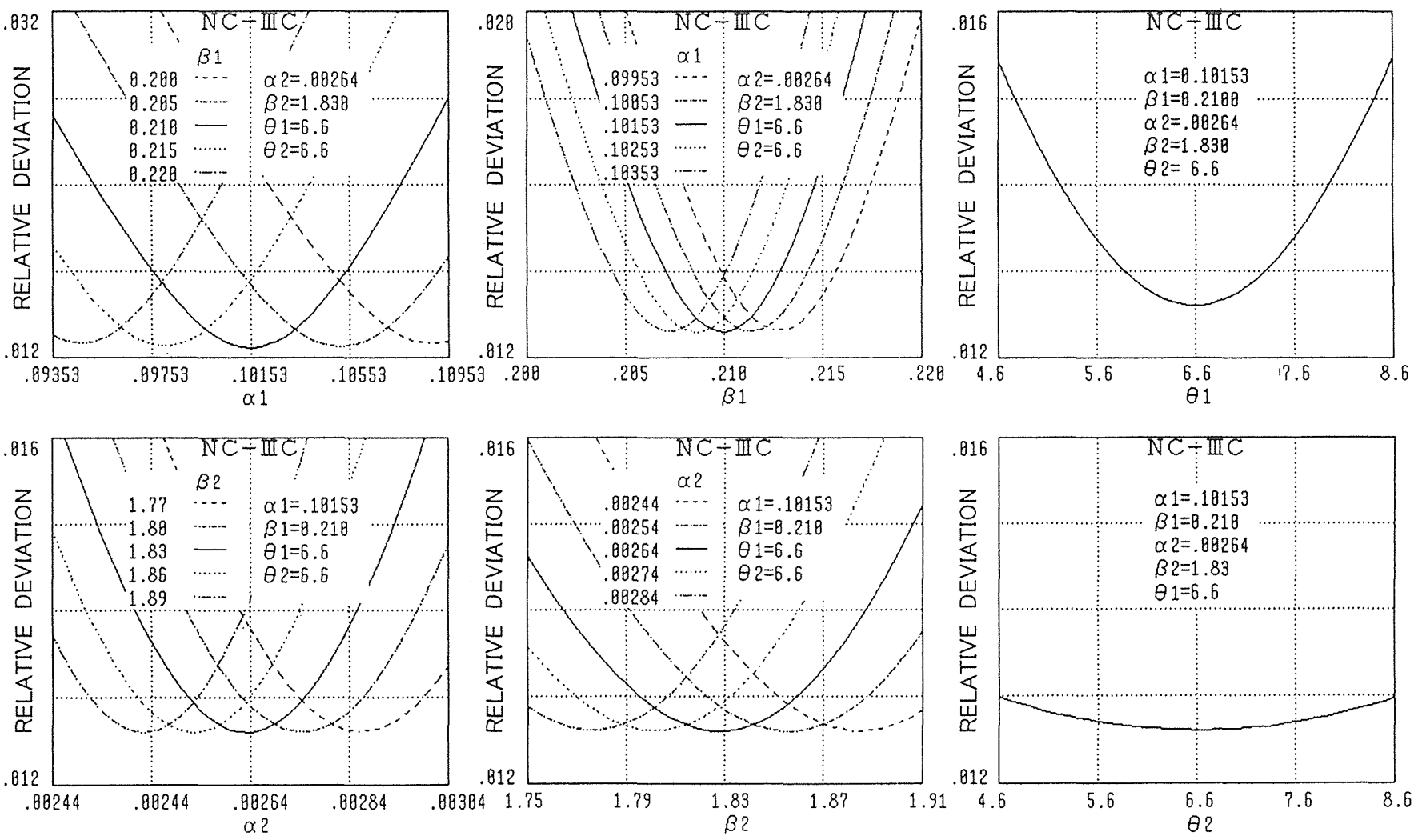

Figure 2 Variation of relative deviation $\sigma$ as functions of coefficients $\alpha_{1}, \beta_{1}, \theta_{1}$ of non-linear function $k_{1}$, and coefficients $\alpha_{2}, \beta_{2}, \theta_{2}$ of $k_{2}$ for non-linear colour space NC-IIC

Table 3 Values of coefficients in non-linear functions $\mathrm{k}_{1}$ and $\mathrm{k}_{2}$ in opponent responses

\begin{tabular}{|c|c|ccc|}
\hline COLOUR SPACE & COEFFICIENT & COEFFICIENT EXPONENT ANGLE \\
\hline $\mathrm{NC}-\mathrm{III} \mathrm{S}$ & $\alpha_{1}=0.09750$ & $\beta_{1}=0.392$ & $\mathrm{p}=4$ & $\theta_{1}=7.0^{\circ}$ \\
$\mathrm{K}=201$ & $\alpha_{2}=0.02100$ & $\beta_{2}=1.353$ & $\mathrm{q}=2$ & $\theta_{2}=7.0^{\circ}$ \\
\hline $\mathrm{NC}-\mathrm{III} \mathrm{C}$ & $\alpha_{1}=0.10153$ & $\beta_{1}=0.210$ & $\mathrm{p}=8$ & $\theta_{1}=6.6^{\circ}$ \\
$\mathrm{K}=255$ & $\alpha_{2}=0.00264$ & $\beta_{2}=1.830$ & $\mathrm{q}=4$ & $\theta_{2}=6.6^{\circ}$ \\
\hline $\mathrm{L}^{*} \mathrm{a}^{*} \mathrm{~b}^{*}-\mathrm{N}$ & $\alpha_{1}=0.06020$ & $\beta_{1}=0.290$ & $\mathrm{p}=8$ & $\theta_{1}=12.0$ \\
$\mathrm{~K}=25 \mathrm{O}$ & $\alpha_{2}=0.00180$ & $\beta_{2}=1.950$ & $\mathrm{q}=4$ & $\theta_{2}=0.0^{\circ}$ \\
\hline
\end{tabular}

of coefficients for non-linear response functions that make relative deviation $\sigma$ the minimum are obtained as shown in Table 3 by respective colour spaces.

Finally, the value of coefficient $K$ is selected so that mean radius of the hue circle of $C=8$ becomes approximately 40 .

The new colour spaces with introduction of non-linearity in opponent responses will be generally named NC-III (New Colour Space-III), and "S" and "C" are put at the end for $1 / 2$ power space and $1 / 3$ power space, respectively. Also, a colour space which is obtained by applying opponent nonlinearity to the colour space $L^{*} a^{*} b^{*}$ will be named $L^{*} a^{*} b^{*}-\mathrm{N}$.

5.2 Characteristics of non-linearity and non-symmetry of opponent colour response functions

It is analyzed what kind of effects the functions $k_{1}$ and $k_{2}$, that show non-linearity and non-symmetry expressed by equations (18) and (22) will bring about to the characteristics of responses in the above spaces. When $k_{1}$ and $k_{2}$ are independently applied to Y-B and R-G responses respectively, standardized outputs $h k_{1}$ and $h k_{2}$ are represented as the functions by variable of opponent colour input $h=\sin \left(\theta-\theta_{1}\right)$ or $h=\cos \left(\theta-\theta_{2}\right)$. These characteristics in respective spaces are shown in Fig.3.

For all colour spaces, the level of non-linearity is larger in the direction of $Y$ than that of $B$ for $Y-B$ response and in the direction of $G$ than that of $R$ for $R-G$ response. In other words, opponent colour responses are non-symmetric and responses of $Y$ and $G$ with higher chroma are more compressed. Moreover, in all colour spaces, levels of nonlinearity and non-symmetry are larger in Y-B response than that of $\mathrm{R}-\mathrm{G}$ response.

\subsection{Opponent colour responses as functions of hue angle}

These opponent colour response functions $k_{1}$ and $k_{2}$ are expressed as the functions by variable of hue angle $\theta$ in Fig.4. It can be observed that compression of $\mathrm{Y}$-B response is larger than that of $\mathrm{R}-\mathrm{G}$ response for all colour spaces.

Values of angles $\theta_{1}$ and $\theta_{2}$, which show the inclination of $k_{1}$ and $k_{2}$ axes corresponding to Y-B and R-G opponent responses, are within a range from $6.6^{\circ}$ to $7.0^{\circ}$ as shown in Table. 3. That is to say, axes of Y-B and R-G responses 

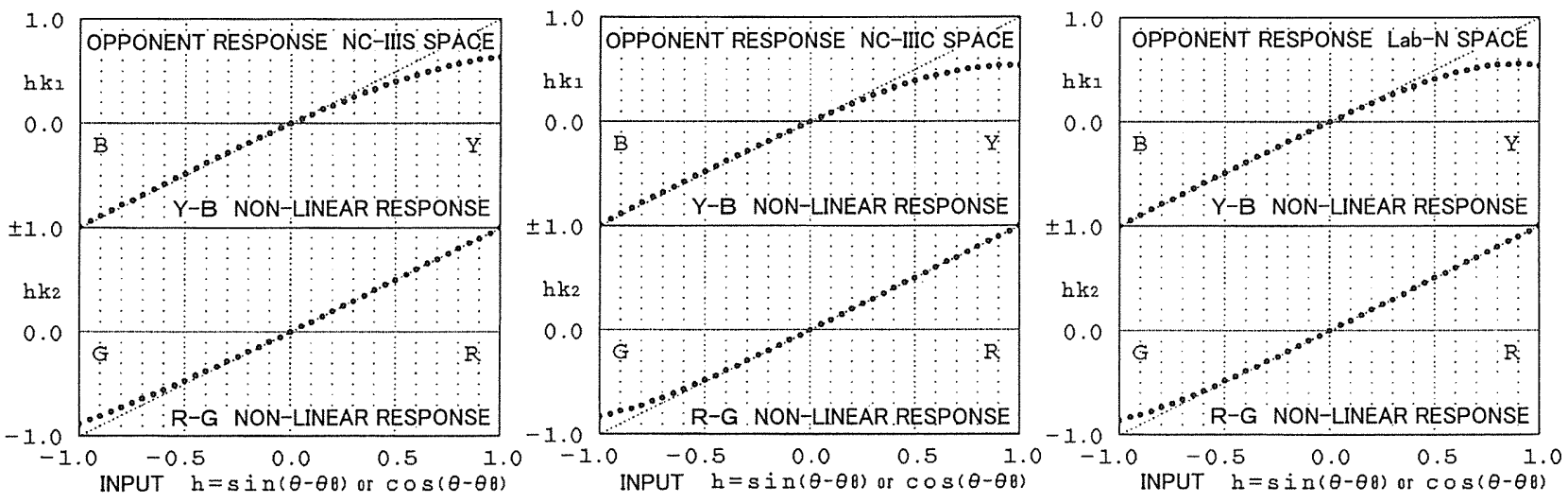

Figure 3 Effects of non-linear functions $k_{1}$ and $k_{2}$ as functions of $h=\sin \left(\theta-\theta_{1}\right)$ or $h=\cos \left(\theta-\theta_{2}\right)$
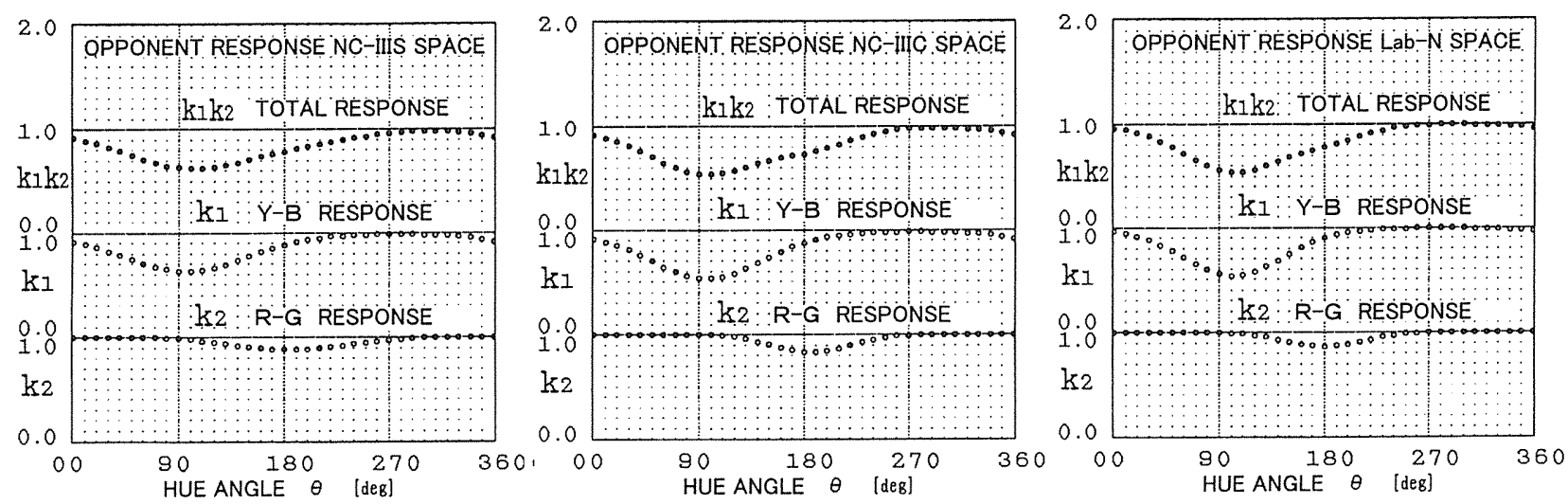

Figure 4 Non-linear opponent response functions $k_{1}, k_{2}$ and $k_{1} k_{2}$ as functions of hue angle $\theta$
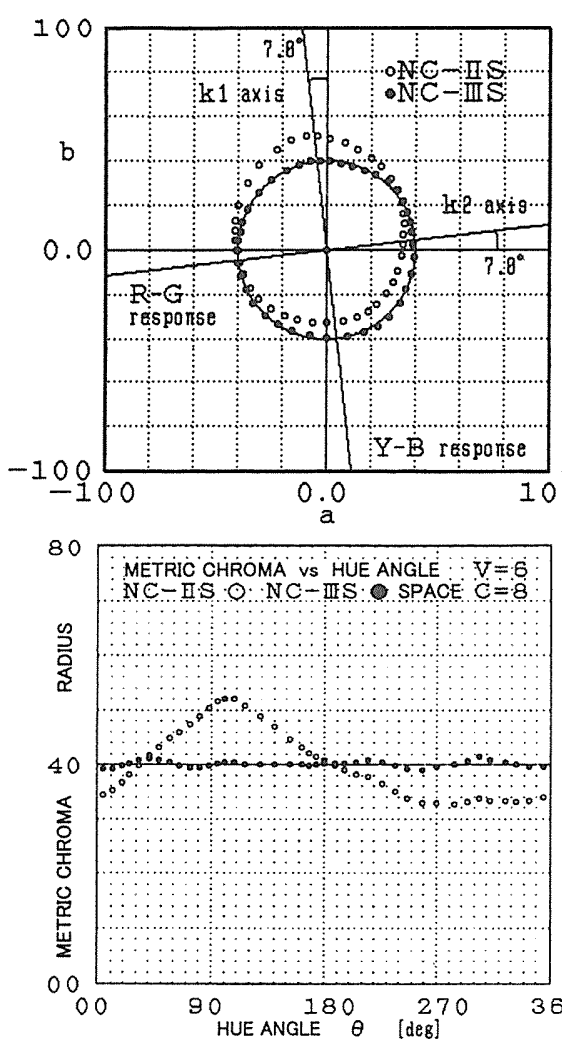

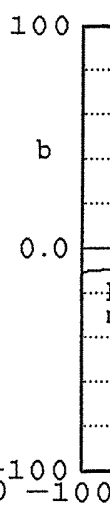

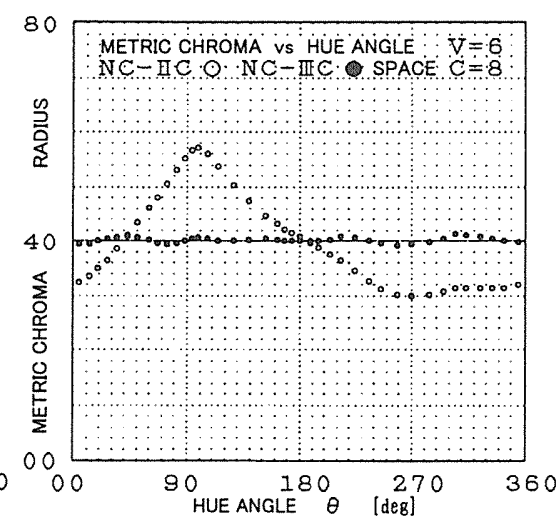

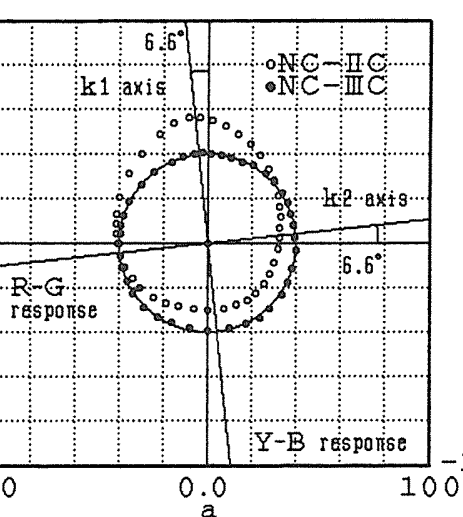

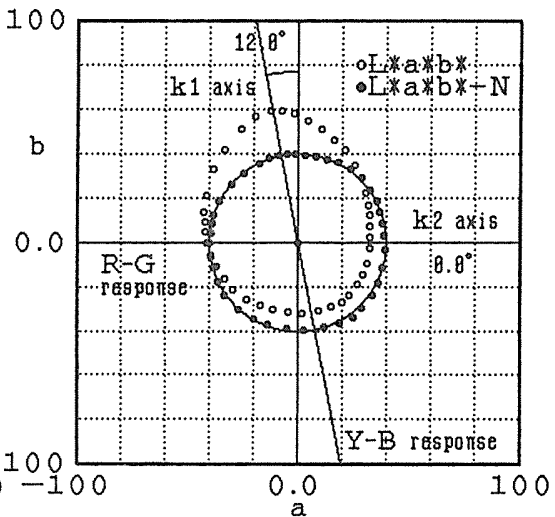

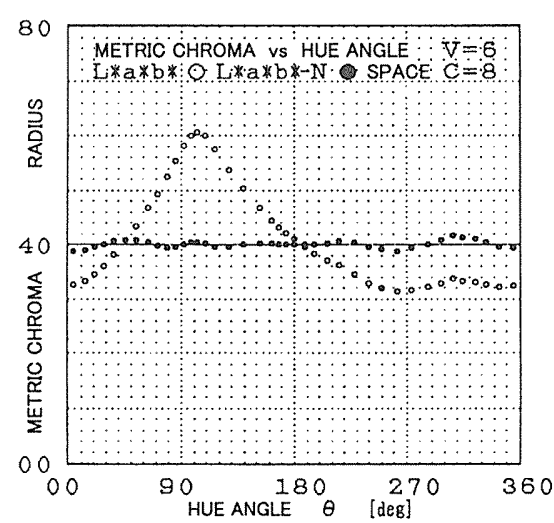

Figure 5 Radial variations of hue circles with value of 6 and chroma of 8 in non-linear colour spaces NC-IIS/NC-IIS, NC-IIC / NC-IIC and L $\mathrm{L}^{*} \mathrm{a}^{*} \mathrm{~b}^{*} / \mathrm{L}^{*} \mathrm{a}^{*} \mathrm{~b}^{*}-\mathrm{N}$ 
are both slightly tilted from coordinate axes of $a^{\prime}$ and $b^{\prime}$ for NC-IIIS and NC-IIIC colour spaces.

However, since $\theta_{1}=\theta_{2}$ is fulfilled for both colour spaces, axes of $k_{1}$ and $k_{2}$ are perpendicular to each other, so it can be regarded that Y-B and R-G responses are independent from the view point of geometrical relations.

On the other hand, as for colour space $L^{*} a^{*} b * \mathrm{~N}$ acquired by applying non-linear and non-symmetric response functions to $L^{*} a^{*} b^{*}$ uniform colour space, angles $\theta_{1}$ and $\theta_{2}$ of $k_{1}$ and $k_{2}$ axes are $12.0^{\circ}$ and $0.0^{\circ}$ respectively, and thus the axes of Y-B and R-G responses are neither independent nor orthogonal to each other.

\section{Mutual comparison of new non-linear colour spaces}

\subsection{Shape of hue circles and variation of metric chroma}

The radial variations of hue circles of Munsell colours with $V=6$ and $C=8$ in non-linear colour spaces NC-IIS / NC-IIIS, NC-IIC/ NC-IIIC and $L^{*} a^{*} b^{*} / L^{*} a^{*} b^{*}=\mathrm{N}$ are shown in Fig.5.

Distance between the coordinates and the origin, in other words metric chroma, significantly varies in any of NC-IIS, NC-IIC and $L^{*}{ }^{*} b^{*}$ uniform colour spaces. The distances in respective new colour spaces which adopt non-linearity and non-symmetry in opponent responses are almost constant, and thus the hue circles of $C=8$ can be represented as uniform circles with same constant radius. Because of this constancy, it becomes possible to correspond the radius with chroma in any of the new colour spaces. Also, the direction of circumference indicating the change of hue and the direction of radius indicating the change of chroma are orthogonal to each other. Therefore it is possible to treat these differential quantities as independent attributes.

Moreover, as clearly confirmed from the shapes and inclinations of hue circles in Fig. 5, Y-B and R-G response axes can be regarded as geometrically orthogonal to each other for NC-IIIS and NC-IIIC spaces. On the other hand, these two axes of opponent responses are not perpendicular for $L^{*} a^{*} b^{*}$ and $L^{*} a^{*} b^{*}$ N spaces.

\subsection{Uniformity of spacing between hue circles}

In addition, to confirm the uniformity of colour specification by non-linear uniform colour spaces in a wide range of chroma, mean radii $\langle R\rangle$ of hue circles and relative deviations $\sigma$ which indicate the degree of deviations of hue circles from uniform circles, in non-linear uniform colour spaces NC-IIIS, NC-IIIC and $L^{*} a^{*} b^{*}$-N for Munsell colours aligned on the plane of $V=6$ and $C=2-14$, are shown in Table 4. Also mean relative deviations $\langle\sigma\rangle$ are compared with those values in $L^{*} a^{*} b$ *uniform colour space.

Up to this point, derivation of NC-IIIS and NC-IIIC nonlinear spaces from linear space NC-I has been made by adjusting coefficients of equations expressing the colour space, so that the hue circle of $C=8$ shapes closer to a uniform circle. As shown in Table 4, hue circles with chroma other than $C=8$ are also ultimately close to uniform circles.
When coefficient $K$ is set to 40 so that radius of the hue circle of $C=8$ becomes five times as much as the chroma, hue circles will be concentric and radii of all hue circles of other chroma become approximately fivefold of respective chroma values with even increment.

Moreover, relative deviations $\sigma$ other than those of $C=8$, are generally remarkably smaller than those in former colour spaces prior to the introduction of non-linear response functions in opponent colour mechanisms. However, the deviation level indicators $\sigma$ between hue circles and uniform circles are generally smaller on $1 / 3$ power space NCIIIC compared to those in other colour spaces.

In NC-IIIS colour space, particularly when chroma is low or high, deviations are approximately twofold greater than those of NC-IIIC. Hence, the uniformity of NC-IIIC space with the 1/3 power type functions is superior.

As described above, compared with NC-IIIS in which distortions of hue circles being large at low and high chroma, the distortions in NC-IIIC space are smaller on average from low to high chroma. This suggests that in expressing nonlinearity at the receptor, 1/3 power type functions are superior to $1 / 2$ power type functions.

Table 4 Mean radii $\langle R\rangle$ and relative deviations $\sigma$ of hue circles with $V=6$ and $C=2-14$ in $L^{*} a^{*} b^{*}$ space and new non-linear colour spaces

\begin{tabular}{|c|c|c|c|c|c|}
\hline \multicolumn{2}{|c|}{ COLOUR SPACE } & $L^{*} a^{*} b^{*}$ & NC-IIIS & NC-IIIC & $L^{*} a^{*} b^{*}-N$ \\
\hline \multirow{5}{*}{ 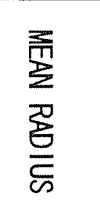 } & \multirow{5}{*}{ 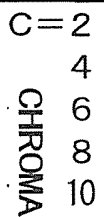 } & 10.339 & 10.052 & 9.919 & 9.890 \\
\hline & & 20.592 & 20.060 & 19. 903 & 19.847 \\
\hline & & 31.142 & 30.320 & 30.228 & 30.145 \\
\hline & & 41.385 & 40.065 & 40.149 & 40.042 \\
\hline & & 51.659 & 49.776 & 50.143 & 50.013 \\
\hline \multirow[t]{2}{*}{$\langle R\rangle$} & 12 & 62.133 & 59. 592 & 60.359 & 60.207 \\
\hline & 14 & 73.901 & 69.619 & 71.382 & 71.209 \\
\hline \multirow{5}{*}{$\frac{\text { 㖇 }}{\frac{\text { 资 }}{\overline{2}}}$} & \multirow{5}{*}{ 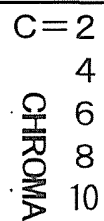 } & 0.2781 & 0.1010 & 0.0688 & 0.0697 \\
\hline & & 0.2430 & 0.0575 & 0.0388 & 0.0402 \\
\hline & & 0.2250 & 0.0203 & 0.0145 & 0.0170 \\
\hline & & 0.2280 & 0.0140 & 0.0126 & 0.0163 \\
\hline & & 0.2222 & 0.0269 & 0.0168 & 0.0204 \\
\hline \multirow[t]{2}{*}{$\sigma$} & 12 & 0.2126 & 0.0485 & 0.0205 & 0.0237 \\
\hline & 14 & 0.2385 & 0.0605 & 0.0316 & 0.0336 \\
\hline$\langle\sigma\rangle$ & MEAN & 0.2288 & 0.0422 & 0.0239 & 0.0266 \\
\hline
\end{tabular}

On the other hand, for $L^{*} a^{*} b^{*}-\mathrm{N}$ space, non-linearity and non-symmetry in opponent processes are introduced likewise in NC-IIIC space, then all hue circles of $V=6$ and $C=2-14$ become concentric circles with equal spacing, and hue and chroma can be represented in a cylindrical coordinate system in correspondence with hue angle and radius of hue circle or metric chroma, respectively.

However, since relative deviations $\sigma$ on $L^{*} a^{*} b^{*} \mathrm{~N}$ space are slightly larger than those on NC-IIC space, it can be said that uniformity of the former is inferior to the latter. 
Also, in this colour space, angles of $k_{1}$ and $k_{2}$ axes in opponent responses are $\theta_{1}=12.0^{\circ}$ and $\theta_{2}=0.0^{\circ}$, respectively, and thus axis of $\mathrm{Y}-\mathrm{B}$ response is inclined from $b^{*}$ axis whereas axis of $R-G$ response is parallel to $a^{*}$ axis. In other words, axes of Y-B and R-G responses are not geometrically orthogonal to each other and not independent.

It can be inferred that the cause of this is due to letting the coefficients for $X$ and $Y$ be whole numbers and that for $Z$ be zero in the expression of $a^{*}$ in order to simplify the expression of $L^{*} a{ }^{*} b^{*}$ uniform colour space.

In contrast, for colour spaces NC-IIIS and NC-IIIC, these coefficients have been adjusted appropriately when developing linear space NC-I, thus the axes of Y-B and R-G opponent responses have been set perpendicular to each other prior to the introduction of non-linear functions.

This indicates that NC-IIIS and NC-IIC spaces are more rational in terms of geometrical structure as the space to specify colour, and hold superior Euclidean geometrical characteristics. All hue circles in both new non-linear colour spaces NC-IIIS and NC-IIIC are approximately concentric, then attributes of hue and chroma can be represented systematically as coordinates in a cylindrical space in correspondence with hue angle and radius of hue circle, respectively.

This result reveals that, despite the observation that there is room for partial improvements to be made on chroma specification in Munsell space through various past studies, generally speaking, the Munsell system is rationally structured with exceptionally superior uniformity.

\subsection{Mutual comparison of hue angles among non-linear uniform colour spaces}

Mean hue angles $\langle\theta\rangle$ of five principal hues with $V=6$ and $C=2-14$, interval $\Delta \theta$ of adjacent hue angles and mean deviations $\sigma_{\theta}$ of hue angles in non-linear uniform colour spaces NC-IIIS, NC-IIIC and $L^{*} a^{*} b^{*}$ N are shown in Table 5 .

Table 5 Mean hue angles $\langle\theta\rangle$ of 5 principal hues with $\mathrm{V}=6$ and $\mathrm{C}=2-14$, intervals $\Delta \theta$ of adjacent hue angles and mean deviations $\sigma_{\theta}$ of these hue angles in new non-linear spaces

\begin{tabular}{|c|c|c|c|c|c|c|}
\hline \multirow{2}{*}{ SPACE } & HUE & $5 R$ & $5 \mathrm{Y}$ & $5 \mathrm{G}$ & $5 \mathrm{~B}$ & $5 P$ \\
\hline & INT & $R-P$ & $Y-R$ & $G-Y$ & $B-G$ & $P-B$ \\
\hline \multirow{3}{*}{$\begin{array}{l}\text { NC-IIIS } \\
\text { [deg] }\end{array}$} & $\langle\theta\rangle$ & 24. 811 & 89.519 & 161.81 & 225.13 & 312.75 \\
\hline & $\Delta \theta$ & 72.065 & 64.708 & 72.287 & 63.321 & 87.619 \\
\hline & $\sigma_{\theta}$ & 0.3594 & 2.5077 & 1.3436 & 3.6110 & 0.9140 \\
\hline \multirow{3}{*}{$\begin{array}{l}\text { NC-IIIC } \\
\text { [deg] }\end{array}$} & $\langle\theta\rangle$ & 25.937 & 88.900 & 162. 34 & 224.16 & 313.76 \\
\hline & $\Delta \theta$ & 72.174 & 62.963 & 73.437 & 61.827 & 89.599 \\
\hline & $\sigma_{\theta}$ & 0.5763 & 2. 2189 & 1.6657 & 2. 6655 & 1.3982 \\
\hline \multirow{3}{*}{$\begin{array}{l}\text { L*a"b"N } \\
\text { [deg] }\end{array}$} & $\langle\theta\rangle$ & 27.665 & 93.006 & 161.52 & 226.86 & 314.11 \\
\hline & $\Delta \theta$ & 73.557 & 65.341 & 68.510 & 65.345 & 87.247 \\
\hline & $\sigma_{\theta}$ & 0.7343 & 2. 3893 & 1.4092 & 3.0808 & 1.1471 \\
\hline
\end{tabular}

Here, the hue angles of these five hues in NC-IIS, NC-IIC and $L^{*} a^{*} b^{*}$ spaces are the same as corresponding hues in respective new colour spaces, because $a^{\prime}$ and $b^{\prime}$ are set so that angles do not change when multiplied by $k_{1}$ and $k_{2}$.

Comparing hue angles among the new colour spaces, hue angles close to $5 \mathrm{Y}$ are 3 to $4^{\circ}$ larger and those close to $5 \mathrm{~B}$ are 2 to $3^{\circ}$ smaller in $L^{*} a^{*} b^{*}$-N space.

This is due to the fact that in $L^{*} a * b^{*} \mathrm{~N}$ space, $k_{1}$ axis indicating Y-B non-linear response in opponent colour response is inclined 5 to $6^{\circ}$ more counter-clockwise and $k_{2}$ axis of $R$-G response is inclined 6 to $7^{\circ}$ more clockwise than other colour spaces.

\section{Coordinates of Munsell colours in non-linear uniform colour space NC-IIC}

Using linear uniform colour space NC-I and Judd linear space as the base, and introducing non-linearities at the receptor level and non-linear and non-symmetric response functions in opponent colour response mechanisms, new nonlinear colour spaces with excellent uniformity, which enable specifications of hue and chroma perpendicularly and independently, have been developed.

Based on a comprehensive judgment of the analyses, it is ascertained that colour attributes can be expressed perpendicularly, independently and with excellent uniformity in new colour spaces that adopt non-linearity in opponent colour response mechanism.

Among these new colour spaces, NC-IIIC space shows the least distortion in hue circles, thus this colour space shall be adopted as the final colour space with the introduction of non-linearity in opponent responses. Coordinates of all Munsell colours of $V=1-9$ in non-linear space NC-IIIC are shown in Fig.6. Mean radii $\langle R\rangle$ of hue circles of each chroma for respective values and mean relative deviations $\langle\sigma\rangle$ which show their gap from uniform circles are indicated in Table 6. For reference, relative deviations $\sigma$ of hue circles of $C=8$ and $V=1-9$ are also shown in Table 6 .

Hue circles of low values of $V=1-3$ are slightly shifted toward 5P, while those of high values of $V=8-9$ are moved toward $5 \mathrm{YG}$. Yet, the cause of shifting is not ascertained.

Naturally, relative deviation $\sigma$ is the smallest when $V=6$ and $C=8$, and relative deviations of hue circles of colours on planes of other values do not exceed over $10 \%$ on the whole. This indicates that the non-linear functions in opponent responses adopted in this study are effective to represent the opponent response mechanisms of the visual system.

In addition, values of hue angles $\theta$, by correspondence with each chroma, of five principal hues of Munsell colours and mean relative deviations $\sigma_{\theta}$ are shown in Table 7 .

Further studies must be conducted based on test data related to perceived hues and colour differences, in order to judge the relevance of specified hue angles. Meanwhile, deviations are generally below $3^{\circ}$ and thus the plotted lines for colours of respective hues are only slightly crooked. 

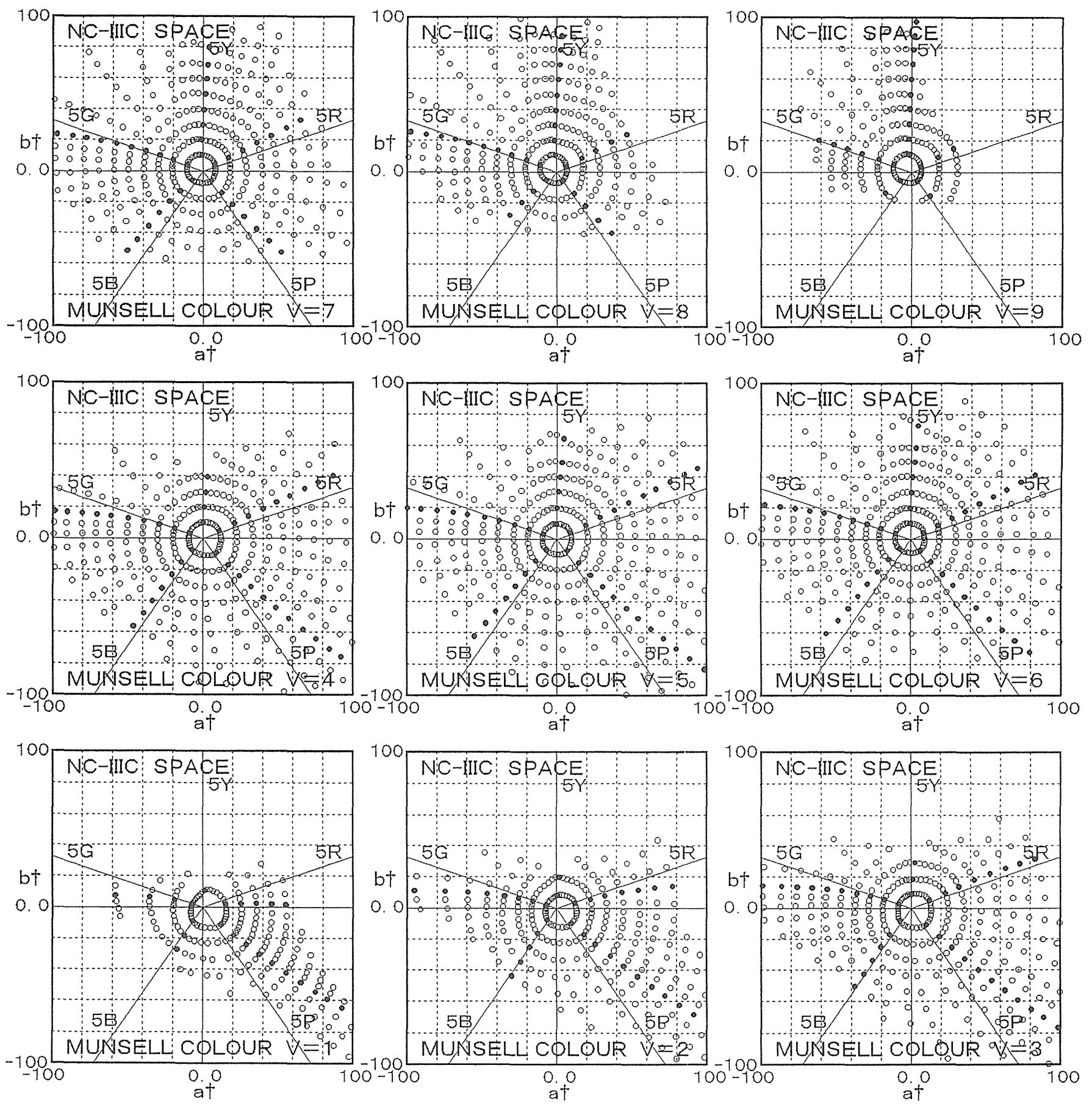

Figure 6 Coordinates of all Munsell colours with values of 1-9 in non-linear uniform colour space NC- IIC

Table 6 Mean radii $\langle R\rangle$ and relative deviations $\sigma$ of hue circles in non-linear uniform colour space NC- IIC

\begin{tabular}{|c|c|c|c|c|c|c|c|c|c|c|}
\hline \multicolumn{2}{|c|}{ NC-IIIC SPACE } & $V=1$ & $V=2$ & $V=3$ & $V=4$ & $V=5$ & $V=6$ & $V=7$ & $V=8$ & $V=9$ \\
\hline \multirow{7}{*}{$\begin{array}{l}\text { 矛 } \\
\text { 芯 } \\
\text { 总 } \\
\langle R>\end{array}$} & \multirow{7}{*}{ 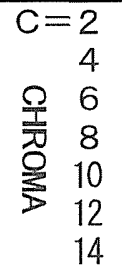 } & 11.945 & 10.349 & 11.209 & 10.779 & 9.967 & 9.919 & 9.587 & 9.167 & 9.408 \\
\hline & & 23. 700 & 20. 779 & 21.990 & 21. 394 & 20. 272 & 19. 903 & 19. 611 & 19. 705 & 21. 083 \\
\hline & & 35.382 & 31.333 & 32. 258 & 31.554 & 30. 705 & 30.228 & 29. 547 & 30. 219 & 32.143 \\
\hline & & 49.067 & 42.568 & 43.324 & 41.935 & 41.116 & 40.149 & 39.779 & 40.935 & 42.807 \\
\hline & & 57. & 53. & 54.197 & $5 ?$ & 51 & 50.143 & 50.270 & 50.824 & 52.643 \\
\hline & & 69.3 & 65.300 & 64. 845 & 63. 403 & 62. 479 & 60.359 & 60.776 & 61.057 & 62. 256 \\
\hline & & 80. 882 & 78.466 & 75.660 & 73. 808 & 72. 164 & 71.382 & 70.626 & 70.269 & 71.657 \\
\hline $\bar{\sigma}$ & $\mathrm{C}=8$ & 0.0962 & 0.0964 & 0.0900 & 0.0479 & 0.0336 & 0.0126 & 0.0144 & 0.0266 & 0.0468 \\
\hline$\langle\sigma\rangle$ & MEAN & 0.0486 & 0.0796 & 0.0921 & 0.0392 & 0.0289 & 0.0239 & 0.0276 & 0.0383 & 0.0453 \\
\hline
\end{tabular}




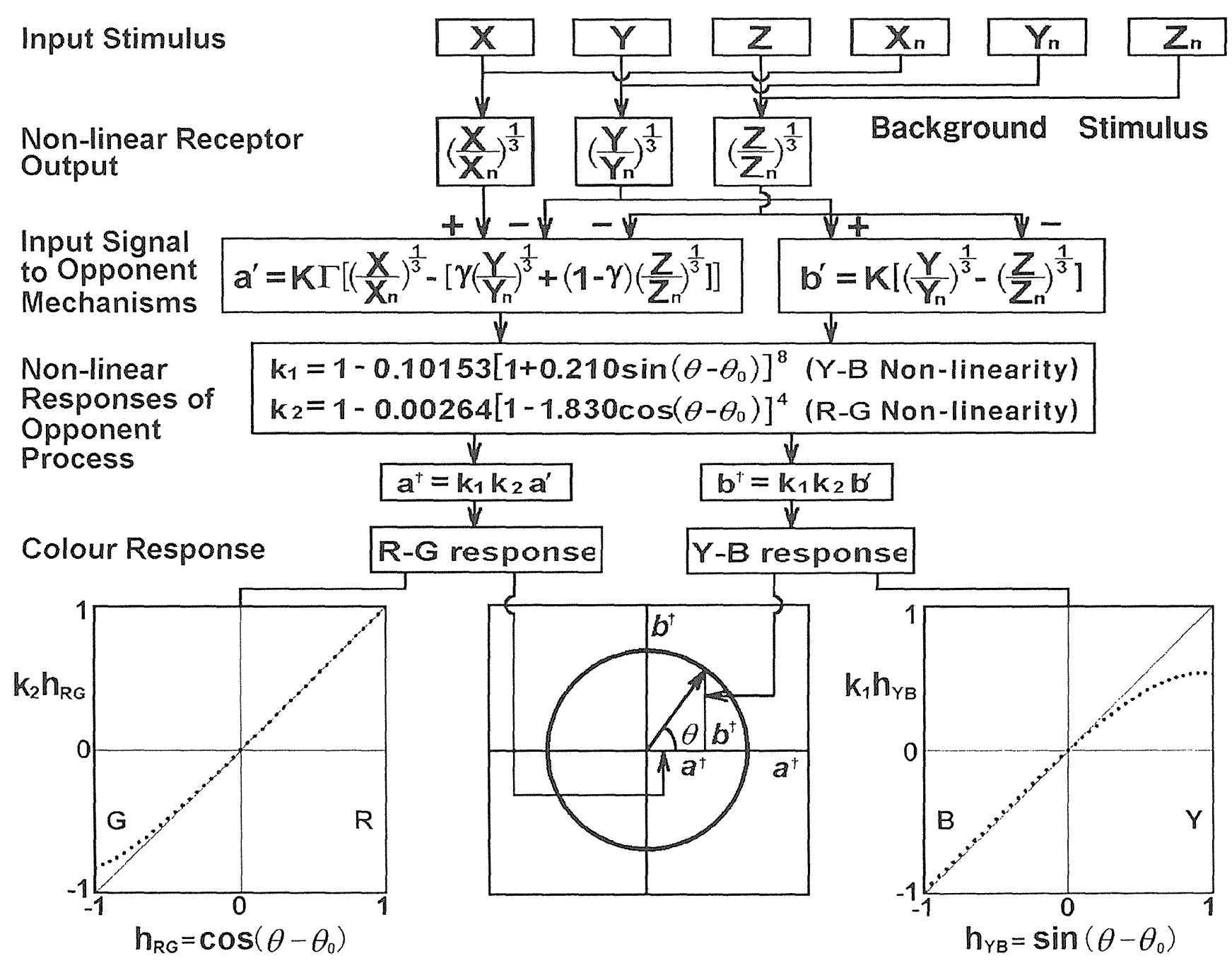

Figure 7 Schematic construction of non-linear and non-symmetric uniform colour space NC- IIIC

Table 7 Hue angles $\theta$ of principal 5 hues with $V=6$ and $\mathrm{C}=2-14$, and deviations $\sigma_{\theta}$ of these hue angles in new uniform space $\mathrm{NC}-$ IIIC

\begin{tabular}{|c|c|c|c|c|c|c|}
\hline \multicolumn{2}{|c|}{ HUE } & $5 R$ & $5 Y$ & $5 G$ & $5 B$ & $5 P$ \\
\hline & $\mathrm{C}=2$ & 24. 664 & 93.014 & 160.03 & 219.55 & 311.60 \\
\hline 鬲 & & 25.616 & 91.214 & 160.73 & 221.36 & 312.43 \\
\hline 3 & & 26. 160 & 89.122 & 161. 29 & 223. 30 & 313.17 \\
\hline ธ & & 26. 192 & 88.026 & 162 & 224. 97 & 313.72 \\
\hline & $\stackrel{?}{\$} 10$ & 26. 341 & 87. 306 & 163 & 226. 14 & 314. 39 \\
\hline$\theta$ & & 26. 274 & 87.021 & 164.07 & 226.78 & 315.17 \\
\hline [deg] & 14 & 26.334 & 86.598 & 164.95 & 227.06 & 315.88 \\
\hline MEAN & $\langle\theta\rangle$ & 25.937 & 88.900 & 162. 34 & 224. 16 & 313.76 \\
\hline DEVI & $\sigma_{\theta}$ & 0.5763 & 2.2189 & 1.6657 & 2. 6655 & 1.3982 \\
\hline
\end{tabular}

At this point, the entire systematic structure image of NCIIIC space, built with the introduction of non-linearity in opponent colour response mechanisms, is shown in Fig. 7 in order to indicate theoretical construction procedures.
The final expressions for NC-IIC space are shown below.

$$
\begin{aligned}
L^{*} & =116(Y / Y \mathbf{n})^{1 / 3}-16 \\
a \dagger & =k_{1} k_{2} a^{\prime} \\
b \dagger & =k_{1} k_{2} b^{\prime} \\
a^{\prime} & =255 \Gamma\left[(X / X n)^{1 / 3}-\left[\gamma(Y / Y \mathbf{n})^{1 / 3}\right.\right. \\
& \left.\left.\quad+(1-\gamma)(Z / Z \mathbf{n})^{1 / 3}\right]\right] \\
b^{\prime} & =255\left[(Y / Y \mathbf{n})^{1 / 3}-(Z / Z \mathbf{n})^{1 / 3}\right] \\
\Gamma= & 2.614040, \gamma=0.974180,(1-\gamma)=0.025820 \\
k_{1}= & 1-0.10153\left[1+0.210 \sin \left(\theta-\theta_{0}\right)\right]^{8} \\
k_{2}= & 1-0.00264\left[1-1.830 \cos \left(\theta-\theta_{0}\right)\right]^{4} \\
\theta= & \tan ^{-1}\left(b^{\prime} / a^{\prime}\right)=\tan ^{-1}\left(b \dagger / a^{\dagger}\right) \\
\theta_{0}= & 6.6^{\circ}
\end{aligned}
$$

Moreover, obtained results can be summarized as follows.

(1) Hue circles can be represented as circles with constant radii, and hue and chroma can be expressed as independent and orthogonal attributes by introducing non-linear functions into opponent response mechanisms. 
(2) It is proved that both non-linearity and non-symmetry in Y-B and R-G opponent colour response mechanisms can be expressed by functions of high degree singular terms.

(3) It became clear that $1 / 3$ power functions are superior to $1 / 2$ power functions in expressing non-linearity at the receptor level, and it is reconfirmed that the concept of non-linearity hitherto adopted in $L^{*} a * b *$ uniform colour space and the like is appropriate.

(4) On NC-IIIC space which is derived from linear colour space NC-I, Y-B response axis and R-G response axis are geometrically perpendicular to and independent of each other. Whereas they are slanted in $L^{*} a^{*} b^{*}-\mathrm{N}$ space derived from Judd linear space. This indicates that the term relating to $Z$ in the equation of $a^{\prime}$ that expresses $\mathrm{R}-\mathrm{G}$ response can not be ignored

\section{Concluding remark}

Up to this point, it has been commonly accepted that physiological colour perceptive mechanisms are constructed by three primary colour responses at the cone level and opponent colour responses at horizontal cell level, respectively, since Young-Helmholtz three-component theory, Hering opponent-colours theory and Hurvich \& Jameson chromatic valence theory. Today, a unified staged theoretical colour perceptive model, which incorporates all of these theories, is widely accepted.

These perceptual responses must be considered non-linear and non-symmetric naturally, because these mechanisms are based on physiological responses. However, with regards to spaces for colour specification, there have been no equations introducing expressions of non-linearity and non-symmetry in opponent colour response mechanisms, while there were equations expressing non-linearity at the cone level

In this study, new non-linear and non-symmetric uniform colour space NC-IIIC for colour specification has been developed successfully by applying non-linearity at the receptor level in linear space and by introducing response functions which express non-linearity and non-symmetry in opponent colour response mechanisms.

As a result, loci or hue circles of colours of constant value and constant chroma in Munsell system can be represented as concentric circles that are placed with even spacing according to the magnitude of chroma.

On reflection, above characteristics of Munsell system are extremely remarkable considering the fact that coordinates of $X, Y$ and $Z$ of Munsell colours were estimated manually in a period when computers were non-existent ${ }^{2}$.

We should pay great respect to this prominent achievement of arranging such lots of coordinates by external and internal insertions of enormous data obtained through psychological experiments in a systematic way by human hand.

Honestly speaking; I reconfirmed the fact that Munsell system is constructed in an ultimately systematic manner, and rediscovered that it is appropriately structured to make up Euclidean uniform colour space.

In this non-linear uniform colour space, hue circles can be represented as uniform circles, and hue and chroma can be independently corresponded with hue angle or metric hue and distance from the origin or metric chroma, respectively. Hence it will be possible to specify colour in correspondence with characteristics of human colour vision.

Moreover, the change in hue and that in chroma can be independently corresponded with the change in hue angle and that of distance from the origin or in radius of hue circle, respectively ${ }^{6)}$. This means the colour difference can be specified in accordance with the attributes of human colour perception.

Likewise in the linear colour space, the expression by the equations can be applied to colour under any illuminant because von Kries type compensation is introduced in the non-linear colour space.

In the future, we plan to verify micro uniformity of the new space, particularly relating to hue angle, by applying this colour space to results of colour difference evaluation tests.

We also wish to make an attempt at elucidating the geometric structure of this colour space by applying the concept of non-linearity and non-symmetry in opponent colour response mechanisms to NCS colour system.

\section{References}

(1) CIE , 1986, " COLORIMETRY - second edition - " Publication CIE, No. 15.2,

(2) Newhall, S.M., Nickerson, D. and Judd, D.B., "Final Report of the O.S.A. Subcommittee on the Spacing of the Munsell Colors " Jour. Opt. Soc. Amer., Vol. 33, pp 385-418, (1943)

(3) Japanese Standard Association, "Colour specification -Specification according to their three attributes"

$$
\text { JIS Z } 8721
$$

(4) Wyszecki, Gunter, "TC-1.3 COLORIMETRY PROGRESS REPORT"

Publication CIE , No. 36, pp 161-172,

(5) IKEDA, K, NAKAYAMA, M. and OBARA, K.

" Comparison of Perceived Colour-Differences of Colour Chips with their Colorimetric ones in the CIE 1976 $\mathrm{L}^{*} \mathrm{u}^{*} \mathrm{v}^{*}$ and the CIE $1976 \mathrm{~L}^{*} \mathrm{a}^{*} \mathrm{~b}^{*}$ Uniform Colour Spaces" Publication CIE , No. 50, pp 83-89, (1980)

(6) IKEDA, K, YAMASHINA, H. and ICHUHASHI, A.

" Colour rendering properties of light sources :

New colour space for evaluation " Lighting Research and Technology , Vol. 28(2), pp 97-112, (1996)

(7) IKEDA, K. and OBARA, K., "Linear Uniform Colour Space Composed of Simple Transformations of Tristimulus Values $X, Y$ and $Z^{\prime \prime} \quad J$. Light and Visual Environment, $\quad$ Vol.25(1), pp 49-59,

(2001) 